\title{
Cervical spine Injury clearance protocol in Polytrauma patients
}

\author{
Dr. Rajesh Ambulgekar ${ }^{1}$,Dr. Pritesh Kothari ${ }^{2}$, Dr Prajyot Kandolkar, ${ }^{2}$ \\ Dr. Rohit Takkar². \\ 1. Prof and H.O.D. Dept of Orthopaedics Dr S.C.G.M.C. Nanded. \\ 2. P.G. students Dept of Orthopaedics Dr S.C.G.M.C. Nanded.
}

\begin{abstract}
It is very common in General Hospital that cervical spine injury is missed in Polytrauma patients at the time of admission. In case where there is no clear indication of cervical spine injury, however, patients still need to be evaluated for cervical spine injuries because an unstable cervical spine injury could to delayed \& result in devastation neurological deterioration. So our purpose of study is to reduce the rate of missed cervical spine injury by proposing the clinical guideline for clearance of cervical spine injury.

Keywords: cervical spine injury, neurodeficit, M.R.I., Polytrauma, protocol.
\end{abstract}

\section{Purpose}

It is very common in General Hospital that cervical spine injury is missed in Polytrauma patients at the time of admission. Which came to notice when patients came after some days with some form of neurological deficit which can be prevented if patients is evaluated properly.

So our purpose of study is to reduce the rate of missed cervical spine injury by proposing the clinical guideline for doing so which is safe, efficient, quick, cost effective and applicable to every general hospital.

\section{Introduction}

Polytrauma patient admitted to hospital may have injury to cervical spine which is not immediately obvious. Because patient is neurological normal, with no neck pain or patient is having distracting pain or patient is unconscious.

In case where there is no clear indication of cervical spine injury, however, patients still need to be evaluated for cervical spine injuries because an unstable cervical spine injury could to delayed \& result in devastation neurological deterioration.

Also in advert use of cervical collar for long duration may cause pressure sore. Which can ultimately lead to infection and need for debridment other reported complication of collar are elevated intra cranial pressure, ventilator associated pneumonias. There is difficulty in airway maintenance, difficulty in central venous access.[1]

So it is wise to clear cervical spine injury as early as possible. Clearance of cervical spine may be straight forward in reliable patients who are alert and awake. But require some form of imaging which include plain X-ray, C.T scan, M.R.I,

Clearance of cervical spine is difficult in unreliable patients who cannot reliably participate in evaluation e.g. intoxicated patients, respiratory or metabolic disturbances, brain injury those patients preferably require imaging for clearance.

\section{Method}

600 polytrauma patients admitted during Jan 2012 to Jan 2013 in emergency department at our institute were evaluated according to protocol based on clinical and radiological finding. Detail history of patient was taken regarding mode of trauma, alcohol intoxication, any neck pain, limb weakness etc.

Patients were evaluated clinically with level of consciousness, neurological deficit, neck pain, neck movement(passive movement avoided as it increases chances the neurological complications). On clinical examination if cervical spine injury was suspected or cannot be ruled out or patient is unreliable then Imaging in form of X-ray (AP view and lateral view or open mouth view) C.T, M.R.I. has been done.

If unconscious then head injury, intoxication, sedation ruled out. Collar application preferred till spine was cleared. Conscious patients evaluated clinically as per protocol.

\section{Result}

600 polytrauma patient (reliable patients 580 and unreliable patients 20 ) admitted in our hospital were evaluated for cervical spine injury which was immediately not obvious. Out of 580 reliable patients, 20 patients had posterior neck tenderness and 8 patients had restricted neck movements. Total 26 reliable patients were evaluated by imaging. Out of that 14 patients had cervical spine injury. All unreliable patients were evaluated 
with C.T scan, and out of that 1 patient had cervical spine injury diagnosed by C.T scan. All the unconscious patients were applied cervical collar till cervical spine was cleared out of that 1 developed pressure sore which was healed on dressing.

None of these patients which were cleared using protocol developed neurological symptoms secondary due to missed cervical spine injury.

\section{Discussion}

Cervical spine injuries have been reported to occur in up to 3\% of patients with major trauma and up to $10 \%$ of patients with serious head injury [2]. The neurologic deficits that are a common consequence of these injuries can result in devastating disability for the patient as well as considerable economic and social burden for society. A missed injury can result in delayed treatment, instability, and possible quadriplegia; therefore, it is critical that physicians responsible for the initial evaluation and treatment should follow proper guideline to rule out cervical spine injury.

While it is tempting to focus on the cervical spine, it is important to assess and clear the entire spinal column. The thoracolumbar spine, while more protected, is at risk in major trauma and must be assessed both clinically and radiologically. Additionally, $5 \%$ of spinal injuries have a second, possibly non-adjacent, fracture elsewhere in the spine.

If the patient has an altered level of consciousness or has received sedative drugs, including opioids, the clinical examination may be unreliable.Similarly, distracting pain from a separate (nonspinal) injury may cause the patient to disregard symptoms from an unstable neck injury. Local pain, tenderness and neurological symptoms or signs (such as segmental weakness, numbness or paraesthesia) must be assumed to indicate a potentially unstable injury. In all these circumstances, it is essential to image the spine before moving the neck. However, clinical examination (short of moving theneck) remains an important part of the assessmentand should not be omitted simply because radiography is indicated.

\section{IMAGING}

\section{Plain radiography}

The lateral view is $83 \%$ sensitive and $97 \%$ specific for detecting cervical spine fracture.Adding an open mouth view and AP view increases sensitivity to nearly $100 \%$. The open mouth view is essential for excluding $\mathrm{C} 1$ arch or $\mathrm{C} 2$ odontoid process fractures. The AP view assesses alignment of uncovertebral joints and spinous processes .Oblique views can be used to assess facet joints, pedicles, and lateral mass, especially at the cervicothoracic junction. If the cervico-thoracic junction cannot be visualized on the lateral view, obtain a swimmer's lateral view or a CT scan.

Obtain a CT scan if the patient has normal x-rays but persistent cervical tenderness and pain. Flexionextension views are not useful in the acute setting.Pain and discomfort preclude adequate motion to assess for ligamentous injuries. Only perform a flexion-extension view on an alert patient under supervision. Flexion with an occult ligamentous injury may precipitate neurologic injury.[3],[4],[5]

\section{CT Scan}

A CT scan is useful for determining the presence and extent of osseous injury. In fact, it is superior to MRI in this situation.In blunt trauma patients, CT scan detects $99.3 \%$ of cervical spine fractures.Injuries in the transverse plane may be missed on axial cuts, ie, odontoid fractures.It is essential to obtain coronal and sagittal reconstructions.[6]

\section{MRI}

MRI is indicated for evaluating neurologic deficits and ligamentous injuries.MRI is superior to CT for demonstrating spinal cord pathology, intervertebral disc herniation, and ligamentous disruption.MRI has limited usefulness as the primary means for initial cervical clearance. The highly sensitive images of MRI show muscular and soft tissue images that do not necessarily correlate with clinical instability.MRI is not necessary if the initial screening CT is negative and the patient does not demonstrate neurologic abnormalities.[7]

CT has evolved to be first line modality in obtunded patient in a trauma setting. If the CT is negative, Anderson supports discontinuing the cervical collar.MRI may find abnormalities even if the CT is negative; however, these abnormalities are not likely to be clinically significant.[8] The American College of Radiology advocates both CT and MRI for clearance of the obtunded patient.

\section{Conclusion}

In our study we found that poly trauma patients with no neurological deficit, no neck pain, or distracting pain may have cervical spine injury which may be missed if proper protocol is not followed \& leads to devastating neurological complication. 
For unreliable patients some form of imaging is always required to rule out cervical spine injury as they cannot actively participate in clinical evaluation.

It is preferable to remove hard cervical collar in trauma patient as early as possible. As in advert use of cervical collar for long duration may cause pressure sore.

Flexion extension views of cervical spine $\mathrm{x}$-rays should be avoided in unconscious patients as it may cause cord injury.

M.R.I is preferable to C.T for identifying ligamentous injury, disc, or other soft tissue injuries in suspected cervical spine injury.

general hospital were M.R.I facility not available C.T. scan of cervical spine can be used to rule out cervical spine injury in inconclusive or unconscious patients.

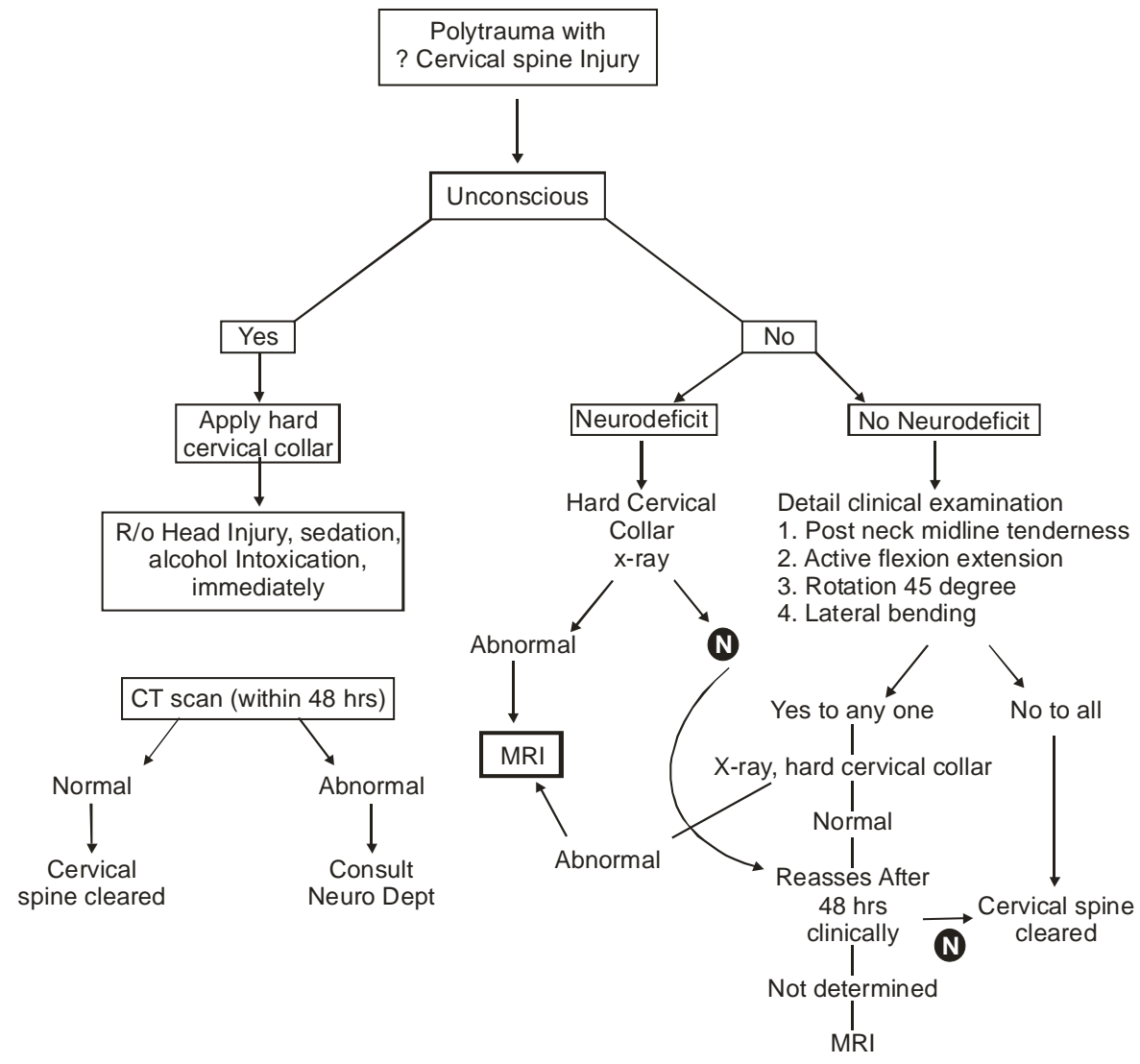

References

[1]. Dunham CM, Brocker BP, Collierr BD, et al. Risks associated with magnetic resonance imaging and cervical collar in comatose, blunt trauma patients with negative comprehensive cervical spine computed tomography and no apparent spinal deficit. Critical Care. 12:R89. Epub, 2008.

[2]. Ajani A., cooper D., Optimal Assessment of Cervical Spine Trauma in Critically ill patients : A prospective Evaluation. J Trauma 1998; 26:487-491

[3]. Stiel IG, Wells GA, Vandemheem KL et al. The Canadian C-Spine rule for radiography in alert and stable trauma patients. JAMA 2001; 286(15):1841-1848.

[4]. MacDonald RL, Schwartz ML, Mirich D, et al. Diagnosis of Cervical Soine injuries in motor vehicle crash victims: how many X-rays are enough? J Trauma 1990; 30(4): 392-397.

[5]. Buckholtz RW et al. Rockwood and Greens Fractures in Adults. Seventh Edition. 2010. Chapter 41- Principles of Spine Trauma Care

[6]. Brown CV, Antevil JL, Sise MJ, Sack DI. Spiral computed tomography for the diagnosis of cervical, thoracic and lumbar spine fractures: Its time has come. J Trauma 2005; 58: 890-895

[7]. Thuret S, Moon LD, Gage FH: Therapeutic Interventions after spinal cord injury. Nat Rev Neurosci 2006:7(8): 628-643

[8]. Hogan GJ, Mirvis SE, Shanmuganathan K, Scalea TM: Exclusion of unstable cervical spine injury in obtunded patients with blunt trauma: Is MRI needed when mutli-detector row CT findings are normal? Radiology 2005; 237: 106-113 